\title{
Molecular Bases of Odor Discrimination: Reconstitution of Olfactory Receptors that Recognize Overlapping Sets of Odorants
}

\author{
Kentaro Kajiya, ${ }^{1}$ Koichiro Inaki, ${ }^{2}$ Motonari Tanaka, ${ }^{1}$ Tatsuya Haga, ${ }^{2}$ Hiroshi Kataoka, ${ }^{1}$ and \\ Kazushige Touhara ${ }^{1}$ \\ ${ }^{1}$ Department of Integrated Biosciences, Graduate School of Frontier Sciences and 2Department of Neurochemistry, \\ Faculty of Medicine, The University of Tokyo, Tokyo 113, Japan
}

\begin{abstract}
The vertebrate olfactory system discriminates a wide variety of odorants by relaying coded information from olfactory sensory neurons in the olfactory epithelium to olfactory cortical areas of the brain. Recent studies have shown that the first step in odor discrimination is mediated by $\sim 1000$ distinct olfactory receptors, which comprise the largest family of G-protein-coupled receptors. In the present study, we used $\mathrm{Ca}^{2+}$ imaging and single-cell reverse transcription-PCR techniques to identify mouse olfactory neurons responding to an odorant and subsequently to clone a receptor gene from the responsive cell. The functionally cloned receptors were expressed in heterologous systems, demonstrating that structurally related olfactory receptors recognized overlapping sets of odorants with distinct affinities and specificities. Our results provide direct evidence
\end{abstract}

for the existence of a receptor code in which the identities of different odorants are specified by distinct combinations of odorant receptors that possess unique molecular receptive ranges. We further demonstrate that the receptor code for an odorant changes with odorant concentration. Finally, we show that odorant receptors in human embryonic kidney 293 cells couple to stimulatory G-proteins such as Goolf, resulting in odorant-dependent increases in cAMP. Odor discrimination is thus determined by differences in the receptive ranges of the odorant receptors that together encode specific odorant molecules.

Key words: olfactory; odorant; receptor; single-cell RT-PCR; calcium imaging; G-protein; cAMP; HEK293
In the olfactory system, sensory information is processed through a series of distinct anatomical structures beginning at the olfactory epithelium in the nose, in which a variety of odorant molecules are detected, and ending at the higher cortical areas of the brain, in which a perception is constructed (Reed, 1992; Buck, 1996; Hildebrand and Shepherd, 1997; Mori et al., 1999; Nakamura, 2000). In peripheral olfactory neurons, a large family of seven transmembrane G-protein-coupled receptors plays a major role in recognizing a broad range of odorant molecules (Mombaerts, 1999a,b; Buck, 2000). The existence of different receptor structures with identical functions across species implicates an evolutionary process that has been aimed at creating diversity to establish the remarkable discriminatory capacity of the chemosensory system (Dryer, 2000).

Odorant stimuli elicit a series of signal transduction events mediated by the odorant receptors expressed on the surface of the

Received March 28, 2001; revised May 14, 2001; accepted May 29, 2001.

This work was supported by a Grant-in-Aid for Scientific Research-C and on Priority Areas (to K.T.) and Research for the Future of Japan Society for the Promotion of Science (to T.H. and H.K.) from the Ministry of Education, Science, Sports, and Culture, by Core Research for Evolutional Science and Technology of Japan Science and Technology Corporation (to T.H.), and by grants from the Ministry of International Trade and Industry (to K.T.). K.T. is a recipient of grants from the Novartis Foundation for the Promotion of Science and from the Tokyo Biochemical Research Foundation. We thank Dr. R. Reed for a chimera cassette, Dr. Y. Shichida for a bovine rhodopsin cDNA, Dr. M. Simon for G $\alpha 15 / 16$, Dr. Y. Fukui for HEK293T cells, T. Hasegawa Co. Ltd. (Tokyo, Japan) for odorant compounds, Dr. T. Shimizu, Dr. D. Saffen, and J. Ito for reading this manuscript, laboratory members for valuable advice, and many other people for encouragement.

The DNA Databank of Japan accession numbers for the sequences described in the paper are AB061228 (mOR-EG) and AB061229 (mOR-EV).

Correspondence should be addressed to Dr. Kazushige Touhara, Department of Integrated Biosciences, Graduate School of Frontier Sciences, The University of Tokyo, Kashiwa, Chiba 277-8562, Japan. E-mail: touhara@k.u-tokyo.ac.jp. Copyright (ㄷ) 2001 Society for Neuroscience $\quad 0270-6474 / 01 / 216018-08 \$ 15.00 / 0$ olfactory neuronal cilia. Gene knock-out studies (Brunet et al., 1996; Belluscio et al., 1998; Wong et al., 2000) suggest that a cAMP cascade, which is comprised of three components, stimulatory G-protein $\alpha$ subunits G $\alpha$ olf, adenylyl cyclase type III, and cyclic nucleotide-gated channels, is dominant in transmitting odorant signals in the olfactory neurons, whereas the role of an inositol-1,4,5-triphosphate $\left(\mathrm{IP}_{3}\right)$-mediated pathway remains unclear (Breer and Boekhoff, 1992; Schild and Restrepo, 1998). Once the olfactory receptors are activated by odorants, the olfactory neurons are then depolarized by cation influx through cyclic nucleotide-gated channels that are activated by cAMP, as well as by $\mathrm{Ca}^{2+}$-activated $\mathrm{Cl}^{-}$channel current (Kurahashi and Yau, 1993; Fring et al., 2000). These processes allow a chemical signal coded by an odorant to be converted to an electronic signal that is transmitted to the olfactory bulb in which additional tuning events occur.

Individual olfactory neurons express one of 1000 of olfactory receptor repertoire in rodents (Ressler et al., 1993; Vassar et al., 1993). Furthermore, the axons of olfactory neurons expressing the same receptor converge onto specific glomeruli (Ressler et al., 1994; Vassar et al., 1994). Thus, the encoding of an odorant determined by the type of innervated receptors in the olfactory epithelium directly reflects the receptive field in the olfactory bulb in which the input signal is further processed to create the specific odor maps. Functional characterization of odorant receptors suggests that the molecular base of odor discrimination is provided by the odorant recognition spectrum of the individual receptors (Zhao et al., 1998; Touhara et al., 1999; Wetzel et al., 1999; Araneda et al., 2000) and that different odorants are encoded by unique sets of odorant receptors (Duchamp-Viret et al., 1999; Malnic et al., 1999). 
The purpose of this study is to examine the encoding of a set of odorants by determining which receptors they activate. We herein describe the strategy by which we functionally identify odorant receptors and examine their ligand specificities to decipher structure-activity relationships through receptor reconstitution studies. We provide experimental evidence for a combinatorial mechanism that would potentially allow discrimination of a variety of odorants by 1000 receptor molecules. Finally, we demonstrate signal transduction pathways that are activated by odorant receptors through stimulatory G-proteins.

\section{MATERIALS AND METHODS}

Odorants and other reagents. Odorant solutions were prepared directly as 1 or $3 \mathrm{~mm}$ stocks in Ringer's solution (in mM: $138 \mathrm{NaCl}, 5.6 \mathrm{KCl}, 2$ $\mathrm{CaCl}$, $2 \mathrm{MgCl}$, 2 sodium pyruvate, 9.4 glucose, and $5 \mathrm{HEPES}, \mathrm{pH} 7.4$ ) and diluted to give the indicated concentrations before the experiments. Eugenol (EG), vanillin, and ethyl vanillin (EV) were kindly provided from T. Hasegawa Co. Ltd. (Tokyo, Japan), and other odorants were obtained from Tokyo Kasei Co. Ltd. (Tokyo, Japan) or Sigma-Aldrich (Tokyo, Japan). Isoproterenol was purchased from Sigma-Aldrich, carbamylcholine from Nacalai Tesque Inc. (Kyoto, Japan), and pertussis toxin from List Biologic (Campbell, CA).

$\mathrm{Ca}^{2+}$ imaging of olfactory neurons. The fura-2-based $\mathrm{Ca}^{2+}$ imaging experiments of mouse olfactory neurons were performed as described previously (Touhara et al., 1999). Briefly, the olfactory neurons were isolated from the olfactory epithelium of 3- to 4-week-old BALB/c CrSlc mice (Japan SLC, Hamamatsu, Japan) on Cell-TAK (Collaborative Research, Bedford, MA)-coated cover glass, and the fura-2-based $\mathrm{Ca}^{2+}$ imaging of odorant responses were recorded with Argus-50 Ca ${ }^{2+}$ imaging processing system (Hamamatsu Photonics, Shizuoka, Japan).

Molecular cloning of odorant receptor genes from single neurons. The odorant receptor gene expressed in single odorant responsive cells was amplified by use of degenerate primers derived from conserved regions in the olfactory receptor family as described previously (Touhara et al., 1999). 5' and 3' rapid amplification of cDNA ends analyses were performed to determine the remaining coding regions from a mouse olfactory epithelium cDNA library. The full-length receptor genes were then obtained by PCR amplification using $P f u$ DNA polymerase (Stratagene, La Jolla, CA), and the primers designed from the 5'- and 3 '-end sequences. The entire open reading frame was sequenced with an ABI373 sequencer (PE; Applied Biosystems, Foster City, CA).

Construction of chimeric receptors. A PCR fragment containing the first 60 nucleotides of the coding region of bovine rhodopsin [amplified from pBK-CMV Rho-tag construct (Krautwurst et al., 1998) kindly provided by Dr. R. Reed (Johns Hopkins University, Baltimore, MD) and a bovine rhodopsin cDNA kindly provided by Dr. Y. Shichida (Kyoto University, Kyoto, Japan)] was digested with MunI and EcoRI and introduced into an EcoRI site in multiple cloning sites of modified pME18S vector (Tanabe et al., 1988) that possesses $\operatorname{SR} \alpha$ promoter. This vector (pME18S-Rho) was used as a cassette to introduce olfactory receptor cDNAs. The mouse olfactory receptor (mOR)-EG cDNA or the MOR23 cDNA was inserted into EcoRI/XhoI sites, which created two additional residues, Glu and Phe, between the rhodopsin-tag and the receptor sequences. The mOR-EV cDNA was inserted into SalI site, creating four additional residues, Glu, Phe, Val, and Asp, between the rhodopsin-tag and the receptor sequences.

Transient transfection of human embryonic kidney 2937 cells. T-antigen expressing human embryonic kidney 293T (HEK293T) cells, derived from HEK293 cells, were grown in DMEM (Nacalai Tesque Inc.) supplemented with $10 \%$ fetal bovine serum (Life Technologies, Gaithersburg, $\mathrm{MD}$ ) in $5 \% \mathrm{CO}_{2}$. Before transfection, cells were seeded onto 35 mm glass-bottomed dishes (Iwaki Inc., Tokyo, Japan) precoated with poly-L-lysine (Sigma-Aldrich). After $24-48 \mathrm{hr}$ incubation at $37^{\circ} \mathrm{C}, 50-$ $70 \%$ confluent cells were transfected with $1.2 \mu \mathrm{g}$ of receptor cDNA, 0.4 $\mu \mathrm{g}$ of pCIS-G $\alpha 15$, and $0.4 \mu \mathrm{g}$ of pCIS-G $\alpha 16$ using Lipofectamine 2000 (Life Technologies). It should be noted, however, that the presence of either $\mathrm{G} \alpha 15$ or G $\alpha 16$ was sufficient in this assay. For cAMP rescue experiments, mouse G $\alpha$ olf cDNA, which was isolated from a mouse olfactory epithelium cDNA library, was cotransfected with an equal amount of pME18S vector to adjust the total amount of DNA transfected. The predicted amino acid sequence of mouse G $\alpha$ olf was identical with that of rat G $\alpha$ olf (Jones and Reed, 1989), except one amino change of Ser70 in rats to Phe70 in mice. The $\mathrm{Ca}^{2+}$ imaging experiments were performed 24-28 hr after transfection. To obtain good $\mathrm{Ca}^{2+}$ responses, it was critical to maintain healthy HEK293T cells and to perform $\mathrm{Ca}^{2+}$ imaging before confluency. Once the cells become confluent, the number of responsive cells starts to decline.

$\mathrm{Ca}^{2+}$ imaging of HEK293T cells. Transfected cells were loaded with 4 $\mu \mathrm{M}$ fura-2 AM (Molecular Probes, Eugene, OR) for $30 \mathrm{~min}$ at $37^{\circ} \mathrm{C}$. Cells were washed in Ringer's solution, and the $\mathrm{Ca}^{2+}$ imaging experiments were performed as described previously (Touhara et al., 1999). Briefly, odorant solutions were applied sequentially to cells for $20 \mathrm{sec}$ with a peristaltic pump at a flow rate of $1.5 \mathrm{ml} / \mathrm{min}$, and fluorescence at $510 \mathrm{~nm}$ by excitation at 340 or $380 \mathrm{~nm}$ with a xenon lamp was measured by use of Argus-HiSCA $\mathrm{Ca}^{2+}$ imaging system (Hamamatsu Photonics). The isoproterenol or carbamylcholine was applied for $5 \mathrm{sec}$. A 3 min interval was left between each odorant application to ensure that cells were not desensitized as a result of the previous application of odorants. To avoid an artificial $\mathrm{Ca}^{2+}$ response of HEK293T cells, it was critical to keep all of the solutions at the same temperature and to minimize the size of bubbles introduced during the exchange of solutions. It should be of note that, in the case of mOR-EV, the dose-dependent $\mathrm{Ca}^{2+}$ increase was difficult to obtain in the same cell because of severe desensitization in HEK293T cells. Thus, we performed separate experiments starting at various odorant concentrations, and the data were first normalized as percentages of responses to carbamylcholine and shown as percentages of responses to $3 \mathrm{~mm}$ odorant.

cAMP assay. The 50-70\% confluent HEK293T cells $\left(3.5 \times 10^{5}\right.$ cells per well in 24-well plate) were transfected with $0.4 \mu \mathrm{g}$ of receptor cDNA by using Lipofectamine2000 (Life Technologies) for Figure 6, $A$ and $B$. For experiments in Figure $6 C, 0.3 \mu \mathrm{g}$ of receptor cDNA, $0.05 \mu \mathrm{g}$ of pCIS-G $\alpha 15,0.05 \mu \mathrm{g}$ of pCIS-G $\alpha 16$, and $0.2-0.8 \mu \mathrm{g}$ of pME18S-G $\alpha$ olf were cotransfected. After $24 \mathrm{hr}$ after transfection, the cells were preincubated in $1 \mathrm{mM} 3$-isobutyl-1-methylxanthine (IBMX) for $30 \mathrm{~min}$, washed with PBS, and exposed to odorants in Ringer's solution containing $1 \mathrm{mM}$ IBMX for $5 \mathrm{~min}$. The cAMP levels were determined by use of an enzyme immunoassay kit for cAMP (Amersham Pharmacia Biotech, Uppsala, Sweden).

\section{RESULTS}

\section{Functional cloning of odorant receptors}

A combination of calcium imaging and single-cell reverse transcription (RT)-PCR techniques allowed for the functional identification of mouse olfactory receptors for a given odorant from single neurons that express predominantly one type of odorant receptor (Malnic et al., 1999; Touhara et al., 1999). To decipher a combination of odorants and receptors, we applied the single-cell cloning strategy to a series of odorant molecules that have different odors but possess some common structural features, such as a spicy smell (EG) and a balsamic aroma (EV). The methodology and the detail of the functional cloning have been described in our previous paper (Touhara et al., 1999). Among the functionally cloned receptors, we decided to focus particularly on two receptors (mOR-EG and $\mathrm{mOR}-\mathrm{EV}$ ) that were isolated from cells that responded to $\mathrm{EG}$ and $\mathrm{EV}$, respectively (Fig. $1 A, B$ ), because they turned out to have $78 \%$ identity at the amino acid sequence level (Fig. 1C). The mOR-EG and mOR-EV are $86 \%$ identical in the transmembrane domains (TMs). In the regions TM3, TM4, and TM5, which have been implicated to be critical for ligand binding, mOR-EG and mOR-EV are $79 \%$ identical. Regarding the mOR-EV, the same receptor gene was isolated from a different olfactory neuron that responded to EV (our unpublished observation).

\section{Functional expression of the cloned receptors}

To characterize ligand specificities of the receptors, we have tested an adenovirus-mediated gene transfer system followed by functional characterization in olfactory neurons (Zhao et al., 1998; Touhara et al., 1999; Araneda et al., 2000) or in primary cultures (Murrell and Hunter, 1999), an approach that has led previously to success in the functional expression of receptors. 
A.
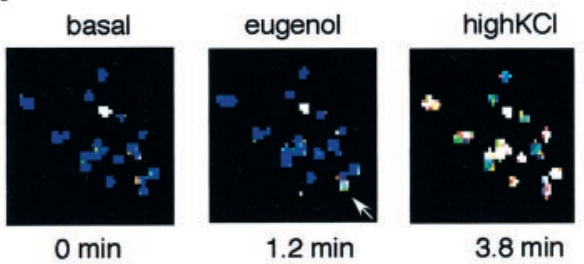

Functional cloning of an olfactory receptor gene expressed in single olfactory neurons that responded to EG or EV. $A$, The $\mathrm{Ca}^{2+}$ response of a single olfactory neuron to EG (shown by an arrow in the top middle panel) in reflected changes in fura-2 fluorescence intensity ratios $(340 / 380 \mathrm{~nm})$. The odorants were applied to olfactory neurons for $4 \mathrm{sec}$ during the times indicated by the black boxes. The cells were washed for $20 \mathrm{sec}$ continuously between odorant applications. Names of odorants are abbreviated as follows: $h 4$, 1-butanol; $l C A$, l-carvone; $L Y$, lyral; $E G$, eugenol; $G E$, geraniol; $E V$, ethyl vanillin; $L I$, lilial; $C R$, cresol; $P Y$, pyridine; $d C A$, Dcarvone; and $B Z$, benzene (each $1 \mathrm{mM}$ ). $H K$ stands for high $\mathrm{KCl}$ buffer. Pseudocolored images of $\mathrm{Ca}^{2+}$ measurements were taken at three representative time points (top panel). White cells indicate high intracellular $\mathrm{Ca}^{2+}$ levels, and blue cells represent the basal level and outlines the shape of each cell. $B$, The $\mathrm{Ca}^{2+}$ response of a single olfactory neuron to EV. $C$, The predicted amino acid sequences of the olfactory receptor genes that were isolated from single olfactory neurons depicted in $A$ and $B$, which correspond to mOR-EG and mOR-EV, respectively. The putative TMs are underlined. In the alignment of the mOR-EG and mOR-EV, residues that differ are highlighted.

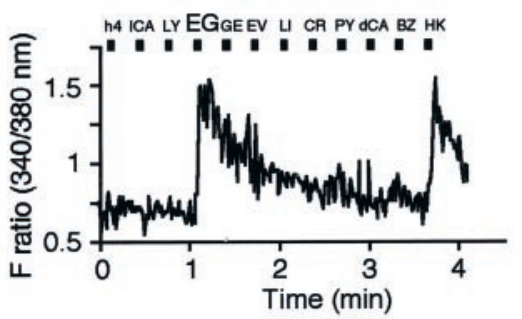

C. TM2 TM4 TM5 TM7
B.
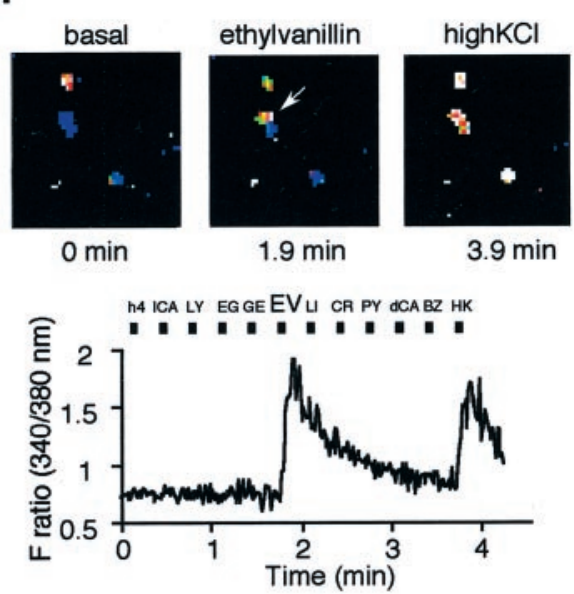

MOR-EG MTLSDGNHSGAVFLLGFSDYPELTIPLFLIFLTIYSITVVGNIGMIVIIRINPRKLHIPMYFFL MOR-EV MILSEKNNSGI IFTLLGF SDYPDLKVPLFLVFLVIYSITVVGNIGMILVIRINPQLHSPMYFFL TM1

MOR-EG SHLSFVDFCYSSIVAPKMLVNLVTMNRGISFVGCLVOFFFFCTFVVTESFLLGVMAYDRFVAIR MOR-EV SHLSFVDFCYSSIIAPKMLVNLVAKD ITISFVECIVOYFLFCVFVVTEA A FLLVVMAYDRFVAIC $\mathrm{TM} 3$

MOR-EG NPLLYTVAMSQRLCAMLVLGSYAWGVVCSLILTCSALNLSFYGFNMINHFFCEFSSLLSLSRSD MOR-EV NPLLYTVAMSQRLCITLVVGSYAWGFTCSLTLTCSTVOLSFHGVNRIDHFFCELSSLLALSSSD

MOR-EG TSVSQLLLFVFATFNE ISTLLIILLSYVLIVVTILKMIKSASGRRKAFSTCASHLTAITIFHGTI MOR-EV TLISQLLLFVFATFNAVSTLLIILLSYLF IVVTVLKMRSASGRRKAFSTCASHLAAITIFHGTI TM6

MOR-EG LFLYCVPNSKNSRHTVKVASVFYTVVIPMLNPLIYSLRNKDVKDTVKKI I GTKVYSS MOR-EV LFLECVPNSKNSRLTVKVGSVFYTVVIPMLNPIIYSLRNKDVQDTIRKIMTLISCVKNDRHN
Unfortunately, no clear odorant response was obtained in these expression systems for unknown reasons (our unpublished observation). We then adopted a heterologous expression system using HEK293T cells along with G-protein $\alpha 15 / 16$ subunits $(\mathrm{G} \alpha 15 / 16)$ that couple to various G-protein-coupled receptors (Krautwurst et al., 1998). The HEK293T $\mathrm{Ca}^{2+}$ imaging assay is an artificial reporter system that can detect receptor activation at the singlecell level with high sensitivity and temporal resolution by forcing the signals to the $\mathrm{IP}_{3}$ pathway by virtue of $\mathrm{G} \alpha 15 / 16$. Viable HEK293T cells showed $\mathrm{Ca}^{2+}$ responses to carbamylcholine via endogenous muscarinic acetylcholine receptors, whereas $\mathrm{Ca}^{2+}$ signals elicited by isoproterenol via endogenous $\beta$-adrenergic receptors were dependent on transiently transfected G $\alpha 15 / 16$ (Fig. 2). A mouse olfactory receptor, MOR23, whose response to the odorant lyral had been recapitulated previously in the adenovirus expression system (Touhara et al., 1999), was tested in the HEK293T expression system. A chimeric MOR23, containing the $\mathrm{N}$-terminal 20 amino acids of rhodopsin, responded to lyral (100 $\mu \mathrm{M})$ and mediated intracellular $\mathrm{Ca}^{2+}$ increases in HEK293T cells (Fig. 2A). Chimeric mOR-EG and mOR-EV were similarly constructed and tested in the same expression system. Figure 2, $B$ and $C$, demonstrates that the mOR-EG and mOR-EV responded to $100 \mu \mathrm{M} E G$ and $1 \mathrm{~mm} E V$, respectively, verifying that the cloned receptors reflect the functional properties of the olfactory neu- rons from which they were isolated. The percentage of odorantresponsive cells was seen in 5-6\% of total cells for the MOR23 (to lyral), $12-13 \%$ for the mOR-EG (to EG), and 2-3\% for the mOR-EV (to EV). No increase in intracellular $\mathrm{Ca}^{2+}$ during ligand stimulations without G $\alpha 15 / 16$ indicates that the MOR23, mOR-EG, and mOR-EV do not couple to endogenous G $\alpha q$ subunits that stimulate an $\mathrm{IP}_{3}$ cascade in HEK293T cells. The $\mathrm{G} \alpha 15 / 16$-transfected cells without the receptors did not respond to the odorants.

\section{Ligand specificity and dose-responses}

To define the receptive range of the mOR-EG, potential agonists were selected by fixing the benzene ring moiety of EG and replacing each of the three functional groups, $-\mathrm{OH},-\mathrm{OCH}_{3}$, and $-\mathrm{CH}_{2} \mathrm{CH}=\mathrm{CH}_{2}$ (chemical structures shown in Fig. 5). The ligand screening of mOR-EG in the HEK293T $\mathrm{Ca}^{2+}$ imaging system yielded responses to vanillin and EV, in addition to EG (Fig. $3 A$ ). Dose-dependent responses of mOR-EG to EG were observed at concentrations of 3-1000 $\mu \mathrm{M}$ (Fig. 3B). The dose-response relationship was fitted to the Hill equation, yielding an $\mathrm{EC}_{50}$ value of $46 \mu \mathrm{M}$ (Fig. 4A). Vanillin, which has an aldehyde group (-CHO) at the position of the allyl group of EG, turned out to be a potent agonist of mOR-EG and exhibited an $\mathrm{EC}_{50}$ value of $36 \mu \mathrm{M}$ (Fig. $3 B, 4 A)$. This value was similar to the $\mathrm{EC}_{50}$ value of $\mathrm{EG}(46 \mu \mathrm{M})$. 
A.
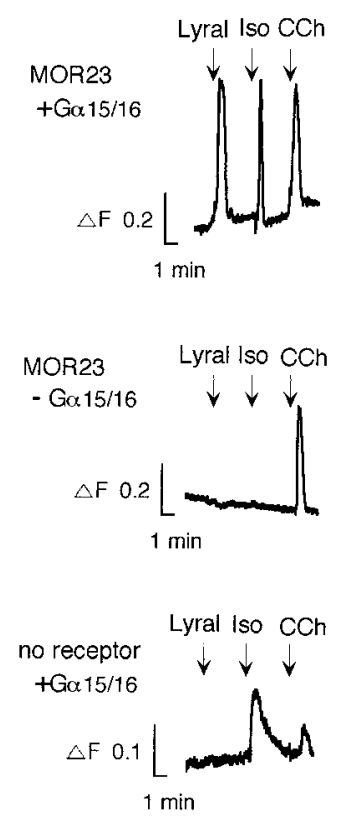

B.
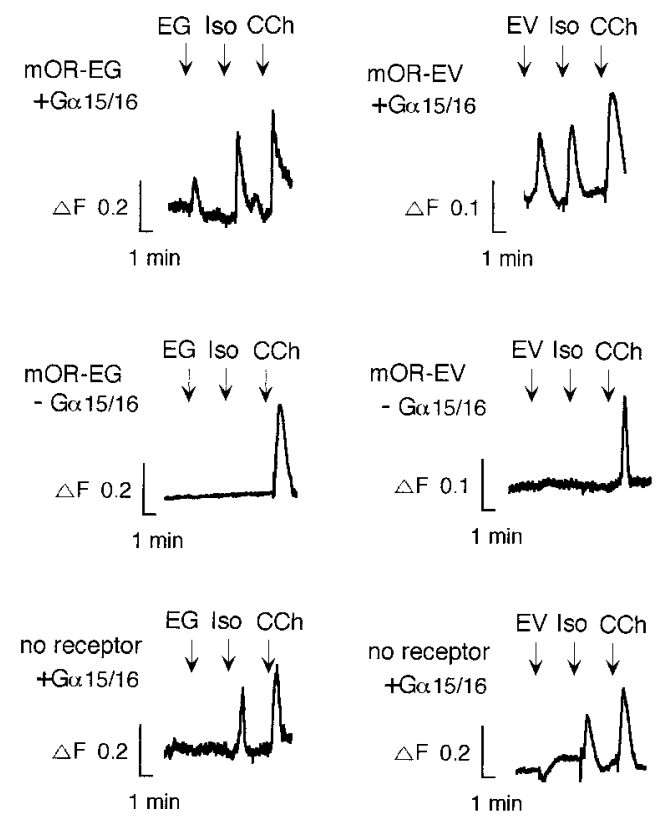

c.

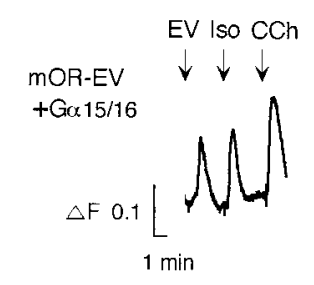

Figure 2. Odorant-induced $\mathrm{Ca}^{2+}$ responses of the functionally cloned receptors in HEK293T cells. The lyral response to the chimeric MOR23 $(A)$, the EG response to chimeric mOR-EG $(B)$, and the EV response to chimeric mOR-EV $(C)$ in fura-2-loaded HEK293T cells cotransfected with the G $\alpha 15 / 16$ subunits. Odorants (lyral and eugenol, $100 \mu \mathrm{M}$; ethyl vanillin, $1 \mathrm{~mm}$ ) were applied for $20 \mathrm{sec}$ at the times indicated by arrows. Carbamylcholine $(C C h)(10 \mu \mathrm{M})$ was applied to verify the viability of cells as a control. Isoproterenol (Iso) $(10 \mu \mathrm{M})$ serves as a control for $\mathrm{G} \alpha 15 / 16$ cotransfection. The middle and bottom panels show responses of cells that were not transfected with $\mathrm{G} \alpha 15 / 16$ (indicated as $-G \alpha 15 / 16$ ) and that were transfected only with $\mathrm{G} \alpha 15 / 16$ (indicated as no receptor $+G \alpha 15 / 16) . \Delta F, \Delta F$ ratio $(340 / 380 \mathrm{~nm})$.
When a substituent at the allyl group was an ethyl or a methyl group (i.e., 2-methoxy-4-ethylphenol (8) or 2-methoxy-4methylphenol (9), respectively), responses were observed at concentrations higher than $300 \mu \mathrm{M}$, suggesting that the mOR-EG has some tolerance for substitution at the allyl group in EG (Fig. 5). The replacement to $-\mathrm{COOH}$ (vanillic acid 6 ) or the removal of the allyl group (guaiacol 2), however, resulted in complete loss of activity. Furthermore, the change in the double bond position in the allyl group, which generates a stereoisomer of EG (isoeugenol 1 ), was also defective in activity, demonstrating that the mOR-EG can distinguish a slight change in the chemical structures (Figs. 3A, 5). Substitution of the hydroxyl group into $-\mathrm{OCOCH}_{3}$ (eugenol acetate 10 ) or $-\mathrm{OC}_{2} \mathrm{H}_{5}$ (eugenol ethyl ether 11) significantly reduced the activity, whereas restriction of the movement of hydroxyl and methoxyl groups by bridging them into an acetal group (safrole 3) or the removal of both functional groups (allylbenzene 4), abolished the activity (Figs. $3 A, 5$ ). EV elicited $\mathrm{Ca}^{2+}$ increases in mOR-EG-transfected cells with an $\mathrm{EC}_{50}$ value $(290 \mu \mathrm{M})$ one order of magnitude lower than those of $\mathrm{EG}$ and vanillin, as shown in the dose-dependent $\mathrm{Ca}^{2+}$ responses (Figs. 3B, 4A).

The mOR-EV recognized EV with an $\mathrm{EC}_{50}$ value of $440 \mu \mathrm{M}$, which was the same order as that of mOR-EG for EV (Fig. 4B). Vanillin was another agonist that elicited $\mathrm{Ca}^{2+}$ responses in the mOR-EV-transfected cells with an $\mathrm{EC}_{50}$ value of $930 \mu \mathrm{M}$, which was approximately twofold lower than the $\mathrm{EC}_{50}$ of EV (Fig. $4 B$ ). This specificity was reversed in the case of the mOR-EG that recognized $\mathrm{EV}$ with a sevenfold lower $\mathrm{EC}_{50}$ than vanillin (Fig. $4 A$ ). These results demonstrated that the mOR-EG and mOR-EV recognized overlapping sets of odorants with different $\mathrm{EC}_{50}$ values. The data also provide direct experimental evidence that a single odorant is recognized by multiple receptors that possess different specificity and that the receptor code for an odorant will change with increasing concentrations. For example, vanillin at $30 \mu \mathrm{M}$ is recognized by a set of odorant receptors, including the mOR-EG but not the mOR-EV, whereas at $1 \mathrm{~mm}$ concentration, the $\mathrm{mOR}-\mathrm{EV}$ participates in the receptor code for vanillin as a vanillin receptor. The mOR-EV did not respond to other odorants used for the mOR-EG ligand screening, although it is possible that other ligands for the mOR-EV exist.

\section{Signaling pathways in HEK293T cells}

Considering the established role of the cAMP cascade in olfactory receptor neurons, one would expect that reconstituted odorant receptors in HEK293T cells also activate stimulatory G-proteins, leading to intracellular cAMP increases. Indeed, when mOR-EG-transfected cells were stimulated by $\mathrm{EG}$, a robust increase in cAMP was observed in a cAMP immunoassay, suggesting that endogenous $\mathrm{G} \alpha$ s coupled with the mOR-EG in HEK293T cells (Fig. 6A). An $\mathrm{EC}_{50}$ value obtained from the dose-response curve of the cAMP assay (59 $\mu \mathrm{M})$ was almost identical with that obtained in the $\mathrm{Ca}^{2+}$ imaging assay (46 $\mu \mathrm{M})$ (Fig. 6B). Other mOR-EG ligands, vanillin and $\mathrm{EV}$, also elicited cAMP increases in the mOREG-transfected cells, whereas other compounds, which were not active in the $\mathrm{Ca}^{2+}$ imaging assay, did not induce cAMP responses (data not shown). The mOR-EV-transfected cells also showed a significant cAMP increase during EV application (Fig. 6A). The lower cAMP increases induced by $\mathrm{EV}$ stimulation in the mOR-EV-transfected cells compared with that by EG stimulation in the mOR-EG-transfected cells is most likely attributable to the difference in the percentages of responsive cells as described previously in Results.

Pretreatment of cells with pertussis toxin did not inhibit the odorant-induced cAMP increases, suggesting that the cAMP increases were not $\mathrm{Gi} / \mathrm{o}(\mathrm{G} \beta \gamma)$-mediated processes (data not shown). Interestingly, the cAMP increases were completely inhibited by cotransfection of $\mathrm{G} \alpha 15 / 16$ that directed the signal to the $\mathrm{IP}_{3}$ pathway (Fig. $6 C$ ). These results suggest that the $\mathrm{G} \alpha \mathrm{s}$ subunits couple odorant receptors, but transfected G $\alpha 15 / 16$ compete with the endogenous $\mathrm{G} \alpha$ s for the receptor coupling. We then cotransfected the stimulatory G-protein $\alpha$ subunits in olfactory receptor neurons, G $\alpha$ olf, and the ratio of transfected G $\alpha$ olf to $\mathrm{G} \alpha 15 / 16$ was gradually increased. As the amount of transfected G $\alpha$ olf was increased, the cAMP response was rescued, demon- 

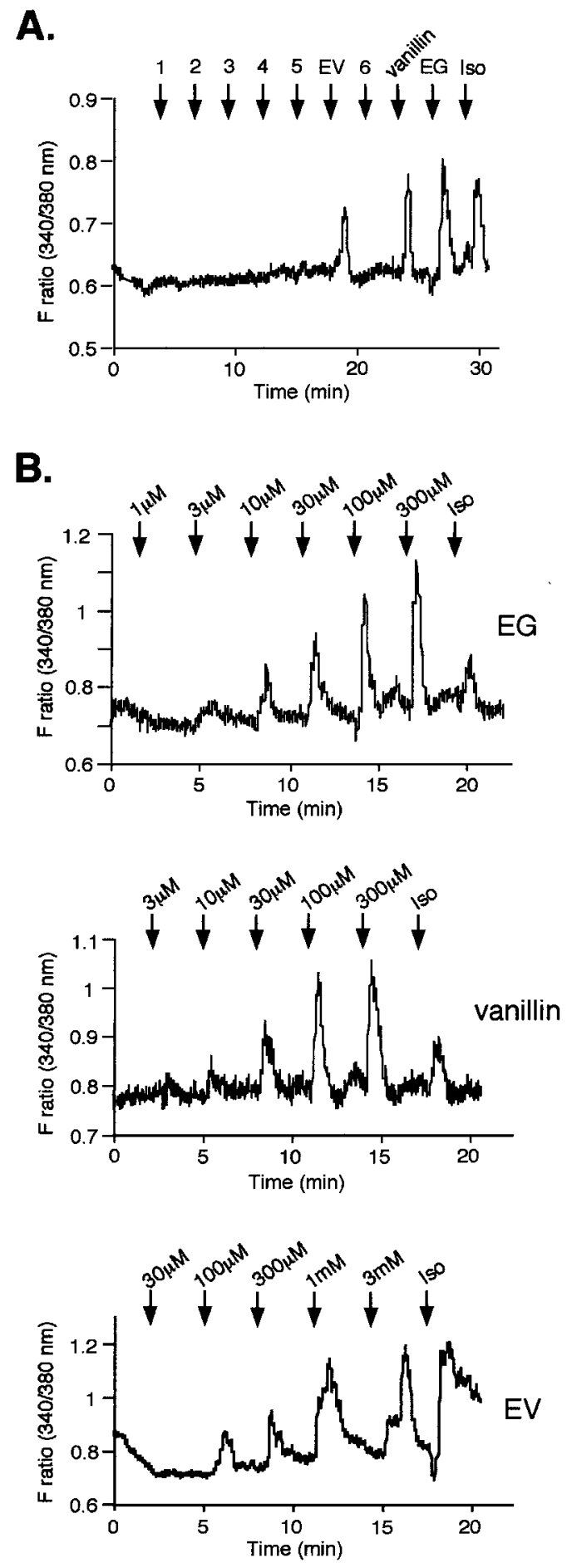

Figure 3. $A$, Odorant responses of the mOR-EG. Various odorants (1 $\mathrm{mM}$ ) were applied for $20 \mathrm{sec}$ at the time indicated by arrows. The mOR-EG shows responses to EG, vanillin, and EV in HEK293T cells cotransfected with $\mathrm{G} \alpha 15 / 16$, as shown by changes in fura-2 fluorescence intensity ratios. Isoproterenol (Iso) $(10 \mu \mathrm{M})$ serves as a control for G $\alpha 15 / 16$ cotransfection. 1, Isoeugenol; 2, guaiacol; 3, safrol; 4, allylbenzene; 5 , syringic aldehyde; 6 , vanillic acid. The structures of these compounds were drawn in Figure 5. B, Dose-dependent $\mathrm{Ca}^{2+}$ responses of the mOR-EG to various ligand odorants. The responses of mOR-EG to increased concentrations of EG, vanillin, and EV in HEK293T cells cotransfected with $\mathrm{G} \alpha 15 / 16$, as shown by changes in fura- 2 fluorescence intensity ratios.
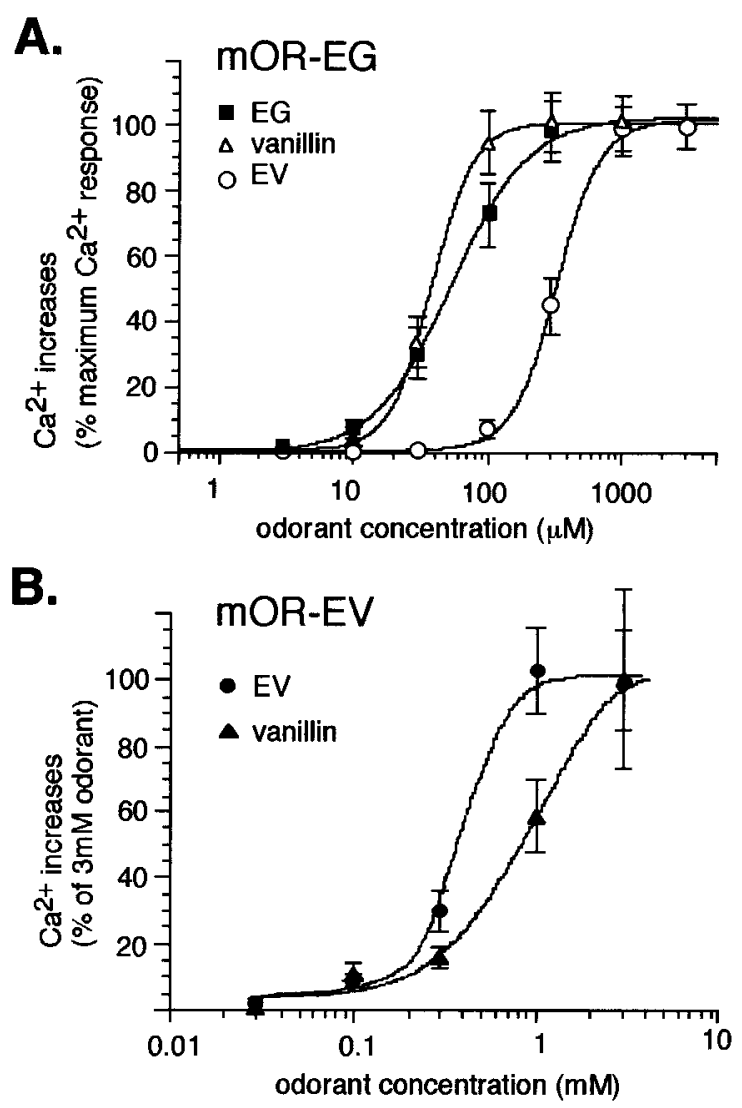

Figure 4. A, Dose-response curves of the mOR-EG to EG, EV, and vanillin, obtained from $\mathrm{Ca}^{2+}$ increases as a percentage of the responses at the highest concentrations of odorants. The maximum responses by ligand odorants were approximately the same. Each point represents the mean \pm SE from at least 20 responding cells. $B$, Dose-response curves of $\mathrm{Ca}^{2+}$ responses of the mOR-EV to EV and vanillin. The data are shown as a percentage of the responses at $3 \mathrm{~mm}$ odorant concentrations. Each point represents the mean $\pm \mathrm{SE}$ from 11-36 cells in three to six separate experiments starting at different concentrations as described in Materials and Methods.

strating the direct G $\alpha$ olf coupling to odorant receptors in the HEK293T reconstituted system (Fig. 6C).

\section{DISCUSSION}

Since the first functional expression studies of a cloned rat olfactory receptor I7 (Zhao et al., 1998), recent accumulated evidence (Krautwurst et al., 1998; Malnic et al., 1999; Touhara et al., 1999; Wetzel et al., 1999) undoubtedly proved that an olfactory receptor originally identified by Buck and Axel (1991) mediated responses to particular odorants that shared specific structural features. The cloning approach to identify retrospectively the odorant receptor gene that individual responsive neurons express resulted in the functional characterization of receptors that specifically recognized a particular odorant of interest (Malnic et al., 1999; Touhara et al., 1999). This strategy allowed for the identification of multiple odorant receptors that recognized the same odorant molecules, which led to the proposal that each particular odorant is encoded by a unique set of odorant receptors and that the olfactory system, therefore, establishes odor discrimination by use of this encoding mechanism in which a combination of activated odorant receptors determine the odor quality (Malnic et al., 1999). Using a reconstituted system, we provided direct experimental evidence for this combinatorial code model in which 


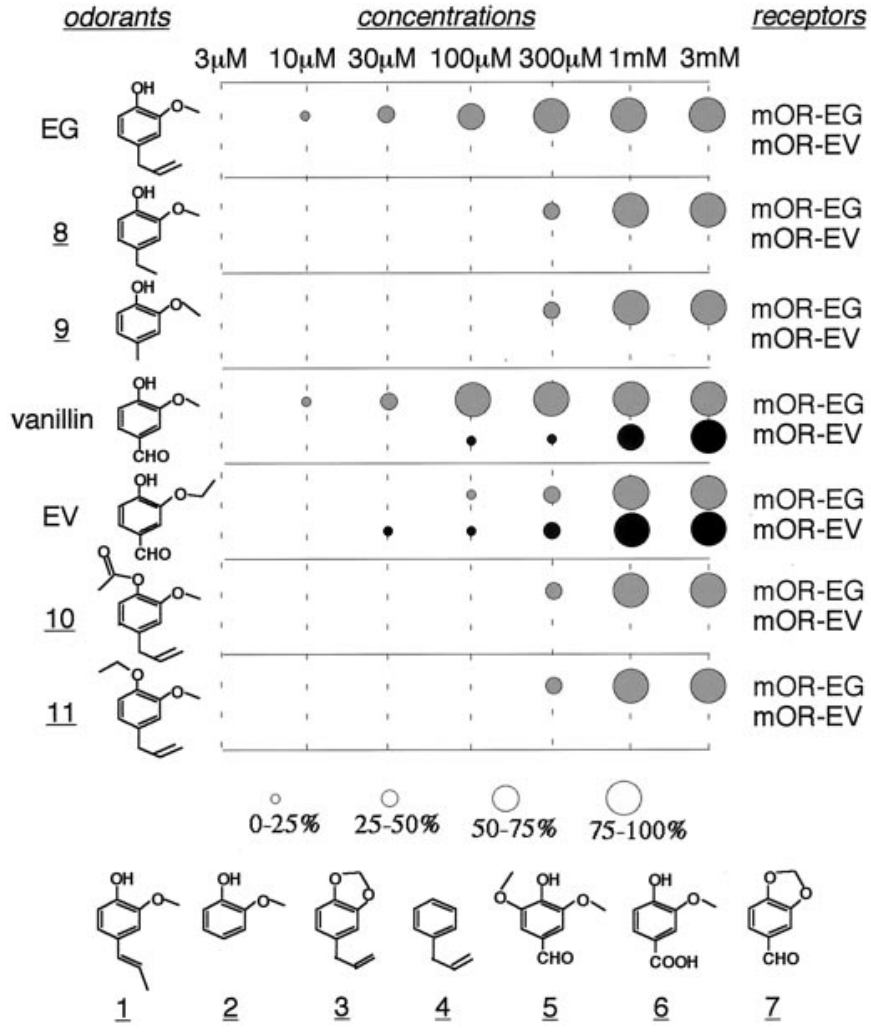

Figure 5. The mOR-EG and mOR-EV recognize overlapping sets of odorants with different affinities and specificities. Dose dependencies of mOR-EG and mOR-EV for various odorant molecules that share some structural similarities with EG or EV were obtained by $\mathrm{Ca}^{2+}$ response assays in HEK293T cells. The sizes of the circles corresponds to the magnitude of $\mathrm{Ca}^{2+}$ responses normalized as a percentage of the responses at $3 \mathrm{~mm}$ ligand: from the smallest circle for $0-25 \%$, to the biggest circle for $75-100 \%$ as indicated. EG and vanillin have the highest affinity for mOR-EG among the odorants tested in this study. The mOR-EG also recognizes odorants $8,9,10$, and 11 at a threshold concentration of 300 $\mu \mathrm{M}$. The mOR-EV recognizes vanillin and $\mathrm{EV}$ with different affinities from those of mOR-EG. Compounds 1-7 did not activate mOR-EG or mOR-EV. 1, Isoeugenol; 2, guaiacol; 3, safrol; 4, allylbenzene; 5, syringic aldehyde; 6 , vanillic acid; 7, heliotropyne; 8, 2-methoxy-4-ethylphenol; 9, 2-methoxy-4-methylphenol; 10, eugenol acetate; 11, eugenol ethyl ether.

structurally related olfactory receptors recognize overlapping sets of odorants with distinct ligand specificities and affinities. We further demonstrated that Goolf was directly coupled to the odorant receptors that recognize cAMP- and/or $\mathrm{IP}_{3}$-elevating odorants.

The rationale for the functional cloning by single-cell RT-PCR technique relies on the belief that a single odorant receptor is expressed per neuron (Ressler et al., 1993; Vassar et al., 1993). However, considering the recent observation that single olfactory neurons sometimes express more than one odorant receptor gene (Rawson et al., 2000), the verification that the cloned odorant receptors reflect the functional properties of the neurons from which they were isolated has to be provided by reconstituting the receptors in functional expression systems. The functional expression of odorant receptors in heterologous systems has been difficult because of the failure of the receptor proteins to be translocated to the plasma membrane in which the responses to odorants occur (Gimelbrant et al., 1999). This difficulty was overcome by the chimeric receptor approach developed by Krautwurst et al. (1998), which allowed efficient protein translo- cation to the plasma membrane by virtue of the $\mathrm{N}$-terminal rhodopsin sequence tag. Similarly, we observed that the flag epitope-tagged chimera mOR-EG, which also showed a specific response to EG, was localized in the plasma membrane area of HEK293T cells as shown by immunocytochemical analyses (data not shown).

The recapitulated responsiveness in the HEK293T cells of mOR-EG and mOR-EV to EG and EV, respectively, supports the notion that a particular odorant receptor expressed in single olfactory neurons reflects the functional properties of the neurons from which they were cloned. The original EG-responsive cell, however, did not show a clear response to $\mathrm{EV}$, whereas the mOR-EG appeared to recognize EV in HEK293T cells, albeit with its lower affinity. This apparent discrepancy is most likely derived from a desensitization mechanism, because EV was applied to the original cell before the EG-mediated $\mathrm{Ca}^{2+}$ signal had completely disappeared (Fig. $1 A$ ).

In some vertebrate species, odorants have been shown to elicit rapid increases in $\mathrm{IP}_{3}$, as well as in cAMP, implicating that two separate signal transduction pathways exist in olfactory neurons (Breer and Boekhoff, 1992; Schild and Restrepo, 1998). The odorants citralva and eugenol appear to increase cAMP, whereas some other odorants such as lyral, lilial, and ethyl vanillin have been shown to elicit $\mathrm{IP}_{3}$ increases in biochemical assays (Breer and Boekhoff, 1992; Schild and Restrepo, 1998). Gene disruption studies of Goolf (Belluscio et al., 1998), adenylyl cyclase III (Wong et al., 2000), and cyclic nucleotide-gated channels (Brunet et al., 1996), however, supported a major role of cAMP in primary odorant responses in the olfactory epithelium. The direct G $\alpha$ olf coupling to odorant receptors, which was shown in this study by the rescue experiment in the HEK293T cells, strengthens the notion that odorant receptors mediate cAMP increases via Goolf coupling. Although both mOR-EV and mOR-EG can recognize ethyl vanillin, which is believed to be an $\mathrm{IP}_{3}$-elevating odorant, these receptors did not elicit $\mathrm{Ca}^{2+}$ signaling without G $\alpha 15 / 16$, suggesting that odorant receptors do not couple to G $\alpha$ q-type subunits in HEK293T cells. We, however, cannot rule out the possible existence of novel types of G-proteins in the olfactory neurons, which couple to odorant receptors and stimulate the $\mathrm{IP}_{3}$ pathway.

Ligand screening performed for mOR-EG revealed that EG and vanillin showed the highest activity in the $\mathrm{Ca}^{2+}$ imaging assay, EV was mid-range, and the compounds $8,9,10$, and 11 depicted in Figure 5 displayed the lowest activity among the odorants tested in this study. Isoeugenol (1), a stereoisomer of EG that has the same molecular weight as EG but has a propenyl group instead of an allyl group, showed no activity for mOR-EG. Together with the fact that the removal of hydroxy and methoxy groups (allylbenzene 4 ) or of the allyl group (guaiacol 2) abolished the activity, the molecular receptive range for an odorant receptor appears to be tolerant but also specific to generate mechanisms to discriminate thousands of odorant molecules and perceive a potentially wide variety of odors. A single olfactory receptor does not necessarily recognize odorants with the same odor, as shown in the case of EG and isoeugenol for the mOR$\mathrm{EG}$, but recognizes ligands based on similarities in molecular structures in a manner similar to other G-protein-coupled receptors.

At the receptor level, an odorant molecule is recognized by a set of odorant receptors that are expected to show structural similarity in amino acid sequence, especially in the TMs. Indeed, the identity between mOR-EG and mOR-EV, which share the 
A.

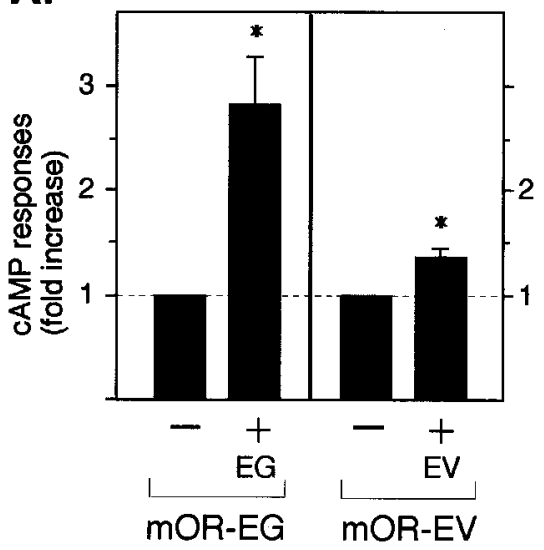

B.

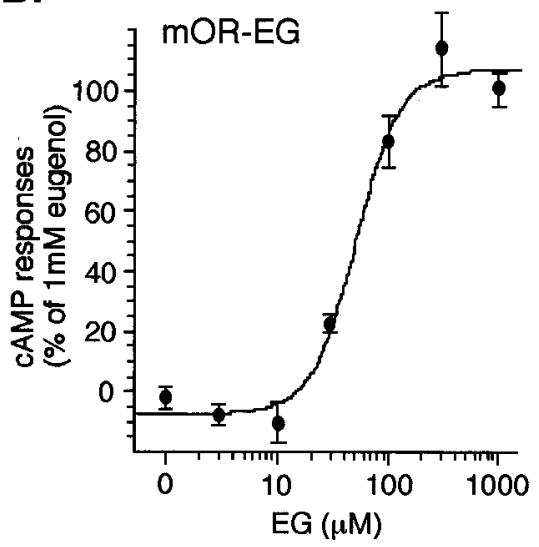

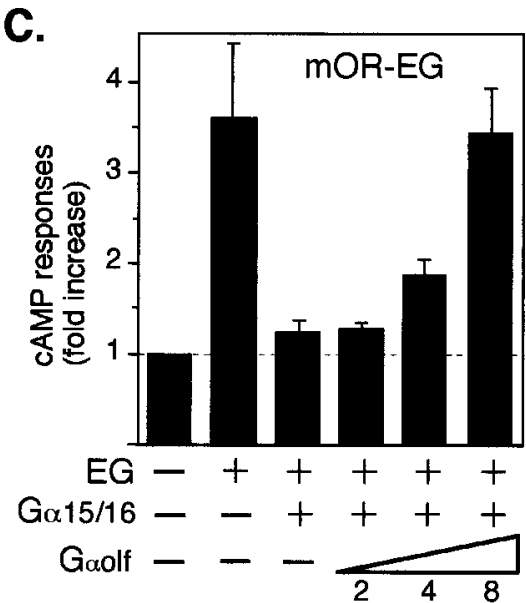

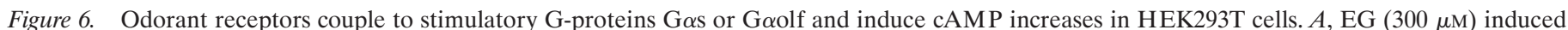

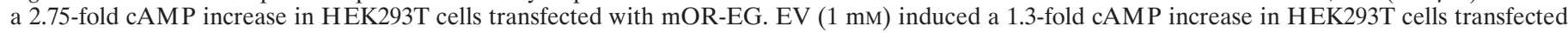

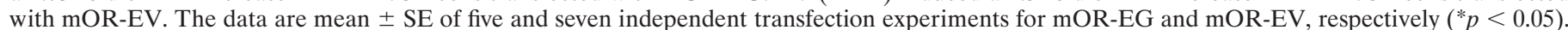

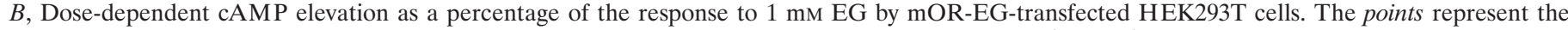

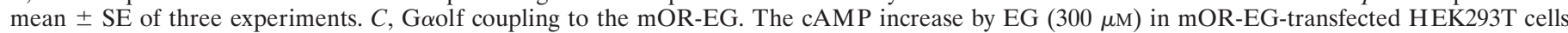

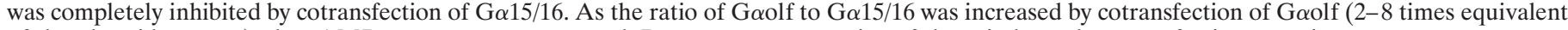
of the plasmid amount), the cAMP responses were rescued. Data are representative of three independent transfection experiments.

ligands, is relatively high considering the overall sequence identity within the family of rodent olfactory receptors (Dryer, 2000). In contrast, other EG receptors, which were identified from cells that responded to EG in our laboratory, were quite diverse in the phylogenic tree (our unpublished observations), suggesting that an odorant is recognized by a diverse set of odorant receptors that include two arrays of receptors: one with high homology at the amino acid sequence level and another exhibiting high sequence diversity. This observation is consistent with the structural relationship in the receptors that were isolated from olfactory neurons that responded to a series of aliphatic odorants with related structures (Malnic et al., 1999). Therefore, it is not easy to predict which odorant receptors are used to encode an odorant from the repertoire of thousands of receptors, based solely on homology at the amino acid sequence level.

The creation of a certain odor or aroma is accomplished by the activation of multiple receptors that lead to the formation of specific activity patterns in the olfactory bulb in which the tuning events occur. Thus, antagonizing or disrupting a single odorant receptor gene, for example, mOR-EG, would not cause anosmia to $\mathrm{EV}$ but may result in a subtle change in overall perception of $\mathrm{EV}$. Another intriguing observation in this study is that mOR-EG and mOR-EV recognized vanillin as a common ligand but with different $\mathrm{EC}_{50}$ values (Fig. 7). Therefore, an odorant appears to be recognized by different sets of odorant receptors at different odorant concentrations, which potentially accounts for our experience that some odorants seem to smell different at different concentrations. Consistent with this notion, recent studies in $\mathrm{Ca}^{2+}$ imaging of intact olfactory epithelia demonstrated that more olfactory neurons responded to higher concentrations of odorant stimuli, presumably because of the presence of receptors that recognize odorant molecules with different affinities (Ma and Shepherd, 2000). Stronger stimuli fire more odorant receptors, which results in a change in the receptor code and, finally, a change in odor quality. This is in contrast to pheromonemediated activation of the vomeronasal organ in which the activation map is independent of concentration (Leinders-Zufall et al., 2000).
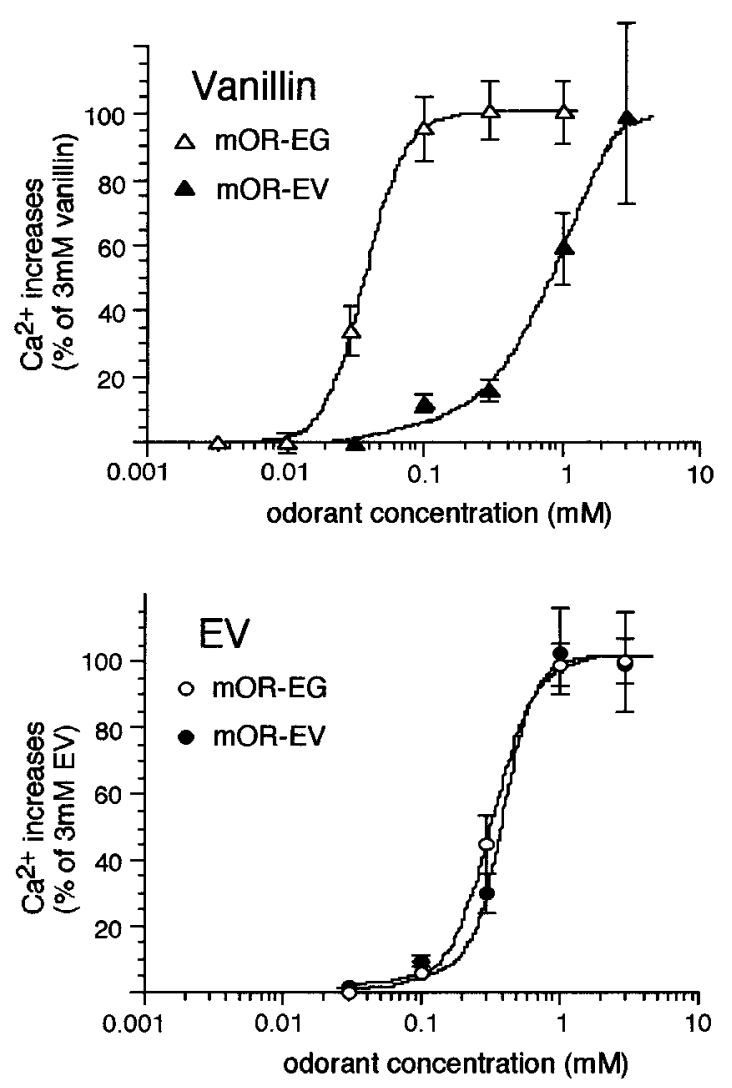

Figure 7. Dose-responses of vanillin and EV for odorant receptors (mOR-EG and mOR-EV). The response curves shown in Figure 4 are redrawn as an odorant-based comparison.

In conclusion, the reconstitution of structurally related odorant receptors that were independently cloned from single olfactory neurons revealed that odorant receptors recognized overlapping sets of odorants with distinct affinities and specificities. The molecular receptive ranges of odorant receptors appear to be highly specific in some aspects but highly nonspecific in other 
aspects. As a result, specific odorants activate a complex combination of odorant receptors. The receptor code for an odorant changes at different concentrations because the encoding of a specific odorant involves multiple receptors, each of which possesses a distinct affinity for the odorant. Finally, evidence for G $\alpha$ olf-mediated increases in cAMP after stimulation of a variety of odorant receptors supports the notion that the cAMP pathway is a common pathway for transmitting odorant signals in the olfactory neurons. Thus, discrimination of odorants takes place at the level of the receptors and not at the level of intracellular signaling. The molecular basis of odor discrimination is determined by the odorant-recognition spectrum of the individual receptors. Activation of specific sets of these receptors determines the pattern of activation in the olfactory bulb, in which the odorant signal inputs from distinct olfactory neurons are further integrated.

\section{REFERENCES}

Araneda RC, Kini D, Firestein S (2000) The molecular receptive range of an odorant receptor. Nat Neurosci 3:1248-1255.

Belluscio L, Gold GH, Nemes A, Axel R (1998) Mice deficient in Golf are anosmic. Neuron 20:69-81.

Breer H, Boekhoff I (1992) Second messenger signalling in olfaction. Curr Opin Neurobiol 2:439-443.

Brunet LJ, Gold GH, Ngai J (1996) General anosmia caused by a targeted disruption of the mouse olfactory cyclic nucleotide-gated cation channel. Neuron 17:681-693.

Buck LB (1996) Information coding in the vertebrate olfactory system. Annu Rev Neurosci 19:517-544.

Buck LB (2000) The molecular architecture of odor and pheromone sensing in mammals. Cell 100:611-618.

Buck L, Axel R (1991) A novel multigene family may encode odorant receptors: a molecular basis for odor recognition. Cell 65:175-187.

Dryer L (2000) Evolution of odorant receptors. BioEssays 22:803-810.

Duchamp-Viret P, Chaput MA, Duchamp A (1999) Odor response properties of rat olfactory receptor neuron. Science 284:2171-2174.

Fring S, Reuter D, Kleene SJ (2000) Neuronal $\mathrm{Ca}^{2+}$-activated $\mathrm{Cl}^{-}$channels-homing in an elusive channel species. Prog Neurobiol 60:247-289.

Gimelbrant AA, Stoss TD, Landers TM, McClintock TS (1999) Truncation releases olfactory receptors from the endoplasmic reticulum of heterologous cells. J Neurochem 72:2301-2311.

Hildebrand JG, Shepherd GM (1997) Mechanisms of olfactory discrimination: converging evidence for common principles across phyla. Annu Rev Neurosci 20:595-631.

Jones DT, Reed RR (1989) Golf: an olfactory neuron specific G protein involved in odorant signal transduction. Science 244:790-795.

Krautwurst D, Yau K-W, Reed RR (1998) Identification of ligands for olfactory receptors by functional expression of a receptor library. Cell 95:917-926.

Kurahashi T, Yau KW (1993) Co-existence of cationic and chloride components in odorant-induced current of vertebrate olfactory receptor neurons. Nature 363:71-74.

Leinders-Zufall T, Lane AP, Puche AC, Ma W, Novotny MV, Shipley MT, Zufall F (2000) Ultrasensitive pheromone detection by mammalian vomeronasal neurons. Nature 405:792-796.

Ma M, Shepherd GM (2000) Functional mosaic organization of mouse olfactory receptor neurons. Proc Natl Acad Sci USA 97:12869-12874.

Malnic B, Hirono J, Sato T, Buck LB (1999) Combinatorial receptor codes for odors. Cell 96:713-723.

Mombaerts P (1999a) Seven transmembrane proteins as odorant and chemosensory receptors. Science 286:707-711.

Mombaerts P (1999b) Molecular biology of odorant receptor in vertebrate. Annu Rev Neurosci 22:487-509.

Mori K, Nagao H, Yoshihara Y (1999) The olfactory bulb: coding and processing of odor molecule information. Science 286:711-715.

Murrell JR, Hunter DD (1999) An olfactory sensory neuron line, odora, properly targets olfactory proteins and responds to odorants. J Neurosci 19:8260-8270.

Nakamura T (2000) Cellular and molecular constituents of olfactory sensation in vertebrates. Comp Biochem Physiol A 126:17-32.

Rawson NE, Eberwine J, Dotson R, Jackson J, Ulrich P, Restrepo D (2000) Expression of mRNAs encoding for two different olfactory receptors in a subset of olfactory receptor neurons. J Neurochem 75:185-195.

Reed RR (1992) Signal pathways in odorant detection. Neuron 8:205-209.

Ressler KJ, Sullivan SL, Buck LB (1993) A zonal organization of odorant receptor gene expression in the olfactory epithelium. Cell 73:597-609.

Ressler KJ, Sullivan SL, Buck LB (1994) Information coding in the olfactory system: evidence for a stereotyped and highly organized epitope map in the olfactory bulb. Cell 79:1245-1255.

Schild D, Restrepo D (1998) Transduction mechanisms in vertebrate olfactory receptor cells. Physiol Rev 78:429-466.

Tanabe Y, Seiki M, Fujisawa J, Hoy P, Yokota K, Arai K, Yoshida M, Arai N (1988) SR $\alpha$ promoter: an efficient and versatile mammalian cDNA expression system composed of the simian virus 40 early promoter and the R-U5 segment of human T-cell leukemia virus type 1 long terminal repeat. Mol Cell Biol 8:466-472.

Touhara K, Sengoku S, Inaki K, Tsuboi A, Hirono J, Sato T, Sakano H, Haga T (1999) Functional identification and reconstitution of an odorant receptor in single olfactory neurons. Proc Natl Acad Sci USA 96:4040-4045.

Vassar R, Ngai J, Axel R (1993) Spatial segregation of odorant receptor expression in the mammalian olfactory epithelium. Cell 74:309-318.

Vassar R, Chao SK, Sitcheran R, Nunez JM, Vosshall LB, Axel R (1994) Topographic organization of sensory projections to the olfactory bulb. Cell 79:981-991.

Wetzel CH, Oles M, Wellerdieck C, Kuczkowizk M, Gisselman G, Hatt H (1999) Specificity and sensitivity of human olfactory receptor functionally expressed in human embryonic kidney 293 cells and Xenopus laevis oocytes. J Neurosci 19:7426-7433.

Wong ST, Trinh K, Hacker B, Chan GCK, Lowe G, Gaggar A, Xia Z, Gold GH, Storm DR (2000) Disruption of the type III adenylyl cyclase gene leads to peripheral and behavioral anosmia in transgenic mice. Neuron 27:487-497.

Zhao H, Lidija I, Otaki JM, Hashimoto M, Mikoshiba K, Firestein S (1998) Functional expression of a mammalian odorant receptor. Science 279:237-242. 